\title{
NIVEL DE INDICADORES DE RESPONSABILIDAD SOCIAL EMPRESARIAL (RSE) EN LA ESE HOSPITAL LOCAL DE MONTELÍBANO, 2015.
}

\section{LEVEL OF INDICATORS OF CORPORATE SOCIAL RESPONSIBILITY (CSR) IN THE ESE HOSPITAL LOCAL DE MONTELIBANO, 2015.}

\author{
Lilibeth Alvarez $C^{1}$, Yurbis Natalia Vásquez F², Enalbis Esther Espitia $C^{3}$ \\ Recibido para publicación: Diciembre 13 de 2016 - Aceptado para publicación: Abril 23 de 2017
}

\begin{abstract}
RESUMEN
Objetivo: Determinar el nivel de los indicadores de (RSE) con el fin de recomendar acciones de mejoramiento en la ESE Hospital Local de Montelibano. Materiales y métodos: Estudio descriptivo transversal cuantitativo, se tomó la población total de los funcionarios directivos, administrativos y operativos. Se utilizó la técnica de la encuesta con aplicación del cuestionario como instrumento para establecer el nivel de responsabilidad social de la empresa desde la percepción de los diferentes grupos de interés como son: Directivos, empleados, proveedores y usuarios determinados en la muestra. Una vez recolectada la información se procedió al registro, procesamiento, análisis y discusión de acuerdo con los objetivos planteados y los resultados de otros estudios usando para ello la elaboración de cuadros estadísticos y técnicas gráficas que ilustraron en forma resumida y organizada la información. La tabulación y graficación se realizó con ayuda de la hoja electrónica de cálculo Excel, la cual permitió hacer operaciones matemáticas sencillas, uso de funciones estadísticas, hasta tablas dinámicas y gráficos. Resultados: el 56\% de la población de los grupos
\end{abstract}

\begin{abstract}
Objective: To determine the level of corporate social responsibility indicators in order to design a strategic plan for improvement in the Local Hospital ESE Montelibano. Materials and Methods: Quantitative Cross-sectional study, the total population of managers, administrative and operational staff took the survey technique was used with application of the questionnaire as a tool to establish the level of social responsibility of the company from the perception of different interest groups such as: Managers, employees, suppliers and users identified in the sample. Once we collected the information we proceeded to the recording, processing, analysis and discussion in accordance with the objectives and results of other studies using for this purpose the preparation of statistical tables and graphs that illustrated techniques summarized and organized information. Tabulation and graphing was performed using electronic Excel spreadsheet, which allowed to make from simple math, using statistical functions, dynamic tables and graphs. Results: $56 \%$ of the population of interest groups officials and users were female, $100 \%$, ages users ranged from 18 to 33 years, and that of officialsin
\end{abstract}

\footnotetext{
${ }^{1}$ Administradora en Salud. Universidad de Córdoba.

${ }^{2}$ Administradora en Salud. Universidad de Córdoba.

${ }^{3}$ Magister en Administración de Organizaciones, Enfermera, Docente Programa Administración en Salud, Universidad de Córdoba Kra. 6 \#76-103 Montería-Córdoba, e-mail:enalbisespitia@correo.unicordoba.edu.co.
} 
de interés, funcionarios y usuarios, era de sexo femenino, $100 \%$ de las edades de los usuarios osciló entre 18 a 33 años, y la de los funcionarios en el intervalo de los 24 a 59 años. En el nivel socioeconómico encontramos que el $89.5 \%$ de los usuarios perteneció al estrato I, mientras que el $8.9 \%$ vivían en estrato II y solo el $1.6 \%$ del total encuestados pertenecieron al estrato III, en los funcionarios se evidenció que la mayoría se clasificaron en un $65 \%$ en los estratos I y II. A nivel general y teniendo en cuenta la valoración de la percepción en cada grupo de indicadores, el nivel de Responsabilidad Social de la institución es de 3,0, la cual la clasificó en un nivel poco satisfactorio. Conclusión: Aunque la Empresa muestra fortalezas en aspectos relacionados con la buena percepción que tienen los grupos de interés frente a factores como el fomento del trabajo en equipo, la no discriminación, la empleabilidad femenina, respeto al derecho de liquidación y despidos, capacitaciones continuas, mecanismos de conocimiento de las expectativas de las comunidades, minimización de desperdicios a través de la realización de programas de auditoría verde; presenta un alto nivel de insatisfacción en aspectos relacionados con el desarrollo de sus empleados y la calidad de vida de las familias y prestaciones superiores a la ley, poco ascenso en empleos femeninos a posiciones ejecutivas, falta de esquemas de trabajos flexibles y de programas para estimular la generación de ideas. Igualmente con la falta de aplicación de normas de certificación ambiental e información detallada del destino de sus productos y o servicios, no perciben metas formales ni declaración de principios medio ambientales para prevenir la contaminación, como tampoco la generación de incentivos previos y reconocimientos para los empleados que sugieran alternativas a los procesos de contaminación. A nivel general y teniendo en cuenta la valoración de la percepción en cada grupo de indicadores, el nivel de Responsabilidad Social de la institución es de 3.0, la cual la clasificó en un nivel poco satisfactorio.

Palabras clave: Responsabilidad social empresarial, Estrategia, Competitividad, Mejoramiento continuo. the range of 24 to 59 years . In the socioeconomic level found that $89.5 \%$ of users belonged to the stratum I, while $8.9 \%$ lived in stratum II and only $1.6 \%$ of the total respondents belonged to the stratum III, officials it became clear that most were classified in $65 \%$ in strata I and II. At a general level and taking into account the assessment of perception indicators in each group, the level of social responsibility of the institution is 3.0 , which classified it in an unsatisfactory level. Conclusion: Although the Company shows strengths in areas related to good perception of stakeholders against factors such as promoting teamwork, nondiscrimination, women's employability, respect for the right of settlement and layoffs, ongoing training, mechanisms of knowledge of the expectations of communities, waste minimization through the realization of green audit programs; It has a high level of dissatisfaction with aspects related to the development of its employees and the quality of life of families and above the law benefits, little rise in women to executive positions jobs, lack of schemes of flexible work and programs to stimulate the generation of ideas. Also with the lack of enforcement of environmental certification standards, and detailed information about the destination of its products or services, they do not receive formal statement or goals of environmental principles to prevent pollution, any generation previous incentives and recognition for employees They are suggesting alternatives to contamination processes. At a general level and taking into account the assessment of perception indicators in each group, the level of social responsibility of the institution is 3.0, which classified it in an unsatisfactory level.

Key words: corporate social responsibility, company, Strategy, Competitiveness, Continuous Improvement. 


\section{INTRODUCCIÓN}

Uno de los temas que más interesa en el mundo académico y empresarial tiene que ver con la RSE (1), ésta es definida como una visión de negocios que integra la gestión de una empresa, teniendo en cuenta los valores y principios éticos, los empleados, la comunidad y el medio ambiente es decir trasciende la obtención de ganancias e incluye la protección y el mejoramiento del bienestar de la sociedad (2). Dentro de los aspectos filantrópicos de la RSE se encuentra las "preocupaciones por el rol del hombre en el desarrollo de la sociedad, las posibles mejoras para sostener el crecimiento económico, las condiciones sociales requeridas para la vida humana, la avaricia humana y sus efectos, entre otros, son ejemplos de cuestionamientos de cada época para fomentar el desarrollo económico y social de cada comunidad" (2).

De acuerdo a lo contemplado por Carnegie (3), se afirma que durante muchos años se desarrolló una visión filantrópica sobre cómo las empresas debían ser más responsables con la sociedad en que participaban [...] bajo esta mirada de la RSE, la preocupación principal estaba basada en la distribución de recursos cuando las empresas obtenían utilidades y no en lo responsable que son las empresas durante el desarrollo de su negocio.

Teniendo en cuenta este punto de vista "La responsabilidad social no se trata de producir y acumular riqueza para después repartirla, setrata de generar riqueza y, al mismo tiempo, remunerar con equidad a todos los factores o actores que intervienen en esa producción de riqueza" (4). Por consiguiente, es necesario contar tanto con el interés propio (de los accionistas) como con el de los otros interesados: trabajadores, clientes, acreedores, proveedores, incluida la sociedad en general. Ante esta situación, las organizaciones deberán tomar estas responsabilidades como vía al desarrollo y éxito empresarial, teniendo en cuenta que deberán ser estructuradas desde un capital humano que ha evolucionado desde un concepto administrativo hasta la consideración del trabajador como un recurso estratégico de tal importancia que, sin su participación, creatividad o entusiasmo, difícilmente podrían desarrollarse las otras funciones básicas de la empresa (5).

Las organizaciones que trascienden los límites de la economía para alcanzar metas a largo plazo que beneficien a la sociedad han decidido ser socialmente responsables. En el caso de la ESE Hospital Local Montelibano en donde se identificó el nivel de Responsabilidad social, los beneficios incluyen facilitar del proceso de toma de decisiones, desarrollo económico, social y competitivo de la empresa, y el bienestar de cada uno de los actores participantes (empleados, comunidad, medio ambiente).

El objetivo principal del proyecto fue determinar el nivel de responsabilidad social con respecto a cuatro dimensiones (calidad de vida de la empresa, ambiente, compromiso con la comunidad, competitividad y relación con los involucrados) (6), a través de un estudio descriptivo, en el que se planteó obtener información primaria usando como técnica la encuesta, e instrumento los cuestionarios elaborados previamente por los investigadores. Se realizó un diagnóstico con el análisis de la información obtenida y se estableció un plan estratégico de mejoramiento que al ser implementado, podría permitirle lograr la competitividad y responsabilidad social en el municipio. El proyecto propendió por tener un impacto ambiental en la medida que la implementación de las estrategias propuestas disminuyan riesgos de contaminación en su contexto; impacto social, cuando se propician actividades de apoyo a la acción social de la empresa en las comunidades e impacto económico que se refleja en la responsabilidad que tiene la empresa de producir los bienes y los servicios que la sociedad necesite, de modo 
que pueda lograr maximizar las ganancias para sus dueños y accionistas. El principio de sustentabilidad aparece y debe ser desarrollado bajo al menos tres ámbitos de desarrollo empresarial: económico, social y ambiental (3), lo cual sustenta y se convierte en el fin del proyecto.

\section{MATERIALES Y MÉTODOS}

Tipo de estudio: se realizó un estudio de tipo descriptivo transversal, con enfoque cualitativo durante el periodo correspondiente a los meses de Julio a Diciembre del año 2015.

Población de estudio: Para llevar a cabo esta investigación se tomó la población total de los funcionarios directivos, administrativos $y$ operativos definidos en la Tabla 1.

Muestra: Para la muestra de usuarios por ser un número mayor se seleccionó la muestra de acuerdo con fórmula estadística, tal como se muestra en la tabla 1.

Para el cálculo del tamaño de la muestra de usuarios se usó la fórmula estadística:

$$
n=\frac{N \times Z_{a}{ }^{2} \times p \times q}{d^{2} \times(N-1)+Z_{a}{ }^{2} \times p \times q}
$$

Donde N=Población; Z=0,95; $p=0,09 ; q=0,1$ y $\mathrm{d}=0,05$. Conuna confianzadel $95 \%$ yun margen de error de 0,09. La muestra de proveedores fue seleccionada por conveniencia.

Criterios de inclusión: se tuvo en cuenta para la selección de la muestra, que los participantes en la investigación cumplieron con los siguientes criterios: Los administrativos y operativos pertenecer a la planta de personal de la institución.

Los proveedores tener más de 1 año de vinculación y encontrarse en la institución al momento de aplicar el instrumento.

Criterios de exclusión: funcionarios que realizan jornadas nocturnas, contratistas de medio tiempo, usuarios de primera vez en la consulta, proveedores ocasionales.

Procesamiento y análisis de la información: Enla recolección de la información los investigadores diseñaron un instrumento basado en diferentes modelos, normas y herramientas existentes y en aplicación tanto en nuestro país como en otras naciones. Para tal efecto se desagregaron la naturaleza y dinámica de una empresa en cuatro áreas fundamentales: Calidad de vida en la empresa, Compromiso con la comunidad, Cuidado y preservación del medio ambiente

Tabla 1. Tamaño de la muestra

\begin{tabular}{lrr}
\hline \multicolumn{1}{c}{ Nombre de la Dependencia } & Población & Muestra \\
\hline Gerencia & 1 & 1 \\
Junta directiva & 5 & 2 \\
Jefes de Unidad (TH, calidad, financiera, sistemas, jefe enfermera) & 6 & 3 \\
Operativos administrativos & 12 & 5 \\
Operativos asistenciales & 19 & 11 \\
Proveedores & 42 & 4 \\
Usuarios & 6200 & 305 \\
Total & & 331 \\
\hline
\end{tabular}


y Competitividad de la empresa y su relación con sus involucrados (stakeholders). Cada una de estas áreas está descrita por un determinado número de indicadores de RSE. Al aplicarla el encuestado estimó en qué medida la empresa cumplía con cada enunciado indicando en una escala del 1 al 5, donde 5 representa el más alto grado de semejanza entre lo que se avaluó y lo que sucedía en la organización, y 1 el más bajo. Una vez recolectada la información se procedió al registro, procesamiento, análisis y discusión de acuerdo con los objetivos planteados y los resultados de otros estudios usando para ello la representación cuantitativa a través de gráficas radar en el que se aplicó la ecuación de la media aritmética para valores que representan la media del indicador a través de la elaboración de cuadros estadísticos y técnica gráfica de radar, los cuales nos ilustran en forma resumida y organizada la información proporcionada por la muestra precisada en el estudio.

\section{RESULTADOS}

Se observó que un $56 \%$ de los encuestados son de sexo femenino tanto en los usuarios como en los funcionarios. De igual manera las edades de los usuarios encuestados van de 18 a 83 años, y la de los funcionarios está en intervalos desde los 24 a 59 años.

Para el nivel socioeconómico encontramos que el $89.5 \%$ de los usuarios pertenece al estrato I, mientras que el $8.9 \%$ vive en estrato II y solo el $1.6 \%$ del total encuestados pertenece al estrato III, en los funcionarios se evidenció que la mayoría representados en un $65 \%$ fueron clasificados en el intervalo de los estratos I y II.

Se evaluaron los indicadores de calidad de vida, de compromiso con la comunidad y de cuidado y preservación del medio ambiente según la percepción a los grupos de interés de la institución representados en los funcionarios, usuarios y proveedores. En general se estableció que el nivel de los indicadores de calidad de vida fue de 3,2 lo que refleja una percepción de poca satisfacción frente a cada uno de losítems evaluados, tal como se ilustra en la Gráfica 1.

\section{INDICADORES CALIDAD DE VIDA}

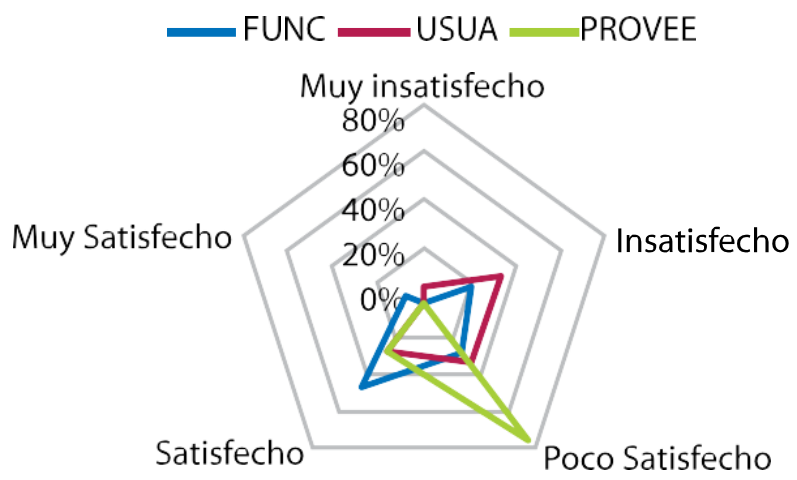

Gráfica 1. Indicadores de calidad de vida

Los miembros de la junta directiva de la empresa manifiestan sentirse satisfechos en un $50 \%$ en aspectos como el fomento del trabajo en equipo, el contar con mecanismos que favorecen el desarrollo de actitudes y habilidades del personal, garantizar la no discriminación por cualquier criterio, maneja respeto, y dignidad en aspectos como liquidaciones y despidos y realiza actividades de capacitación de mejoramiento continuo.

De igual manera el $100 \%$ de los miembros de la junta manifiestan poca satisfacción en cuanto a la realización de investigaciones de clima organizacional para medir el nivel de satisfacción de sus colaboradores e identificar áreas que requieren atención y programas de estimulación y reconocimiento al personal. Manifiestan poca satisfacción, insatisfacción o mucha insatisfacción en aspectos relacionados con: falta de un plan para el desarrollo de sus empleados, calidad de vida de las familias y prestaciones superiores a la ley, poco ascenso en empleos femeninos a posiciones ejecutivas, falta de esquemas de trabajos flexibles y de programas para estimular la generación de 
ideas, decisiones y creatividad y poco estímulo a las oportunidades de trabajo voluntario para los empleados. Los funcionarios encuestados se encuentran distribuidos en dos áreas; elárea administrativa y el área asistencial, en la que se encuesto las auxiliares de enfermería de los diferentes servicios.

La opinión que los funcionarios del área administrativa tienen del indicador de calidad de vida en la empresa, muestra su mayor satisfacción en un $22 \%$ en aspectos como: garantiza políticas de asociación, el incremento de número de empleos femeninos, manejan con respeto el derecho a liquidaciones y despidos y se preocupa por realizar capacitaciones continuas a los empleados.

Igualmente se encuentran satisfechos en un $66.7 \%$ en aspectos relacionados con el fomento del trabajo en equipo, implementación de mecanismos para escuchar las sugerencias y reclamaciones y favorecimiento del desarrollo de actitudes a través de la capacitación, aplicación de prácticas laborales que favorecen el equilibrio entre el personal y las familias y la existencia de normas escritas que prohíben y prevén medidas disciplinarias contra prácticas discriminatorias entre empleados. En contraste se manifiestan poca satisfacción en lo relacionado con la disposición de realizar investigaciones de clima organizacional $y$ desarrollar campañas periódicas para informar e identificar áreas que requieran atención.

Manifiestan insatisfacción en aspectos relacionados con la oferta de oportunidades de trabajo voluntario para sus empleados en proyectos sociales, no contar con programas para estimular y reconocer al personal por la generación de ideas y no se cuenta con un programa especial para inclusión y contratación de grupos específicos. Por otro lado los proveedores perciben que el nivel de satisfacción es del $75 \%$ en cuanto a que la empresa garantiza con sus políticas la no discriminación por ningún motivo de origen étnico, género, o posición social, etc.

En cuanto a los indicadores de compromiso con la comunidad, en general se estableció que el nivel de los indicadores de compromiso con la comunidad fue de 3,3 lo que refleja una percepción de poca satisfacción frente a cada uno de los ítems evaluados, tal como se ilustra en la Gráfica 2.

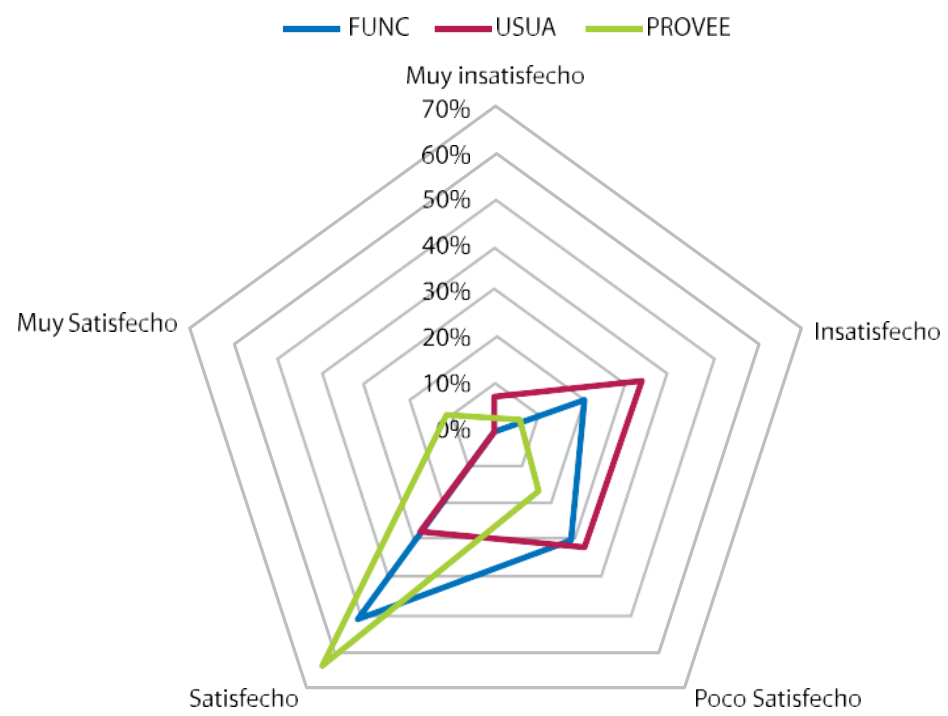

Gráfica 2. Indicadores de compromiso con la comunidad 
De acuerdo con los indicadores de compromiso la comunidad y la junta directiva manifiesta total satisfacción $(100 \%)$ en relación con las expectativas y necesidades de la comunidad, estableciendo canales de comunicaciones entre sí en un 50\%; el porcentaje en mención involucra a las familias y promueve el trabajo con voluntario, buscando oportunidades para promover productos $\mathrm{y} / \mathrm{o}$ servicios $\mathrm{y}$, adoptan medidas necesarias para garantizar las actividades sobre la comunidad.

Asímismo, según la opinión de los funcionarios, estos se encuentran satisfechos con las respuestas a la expectativa que la comunidad tiene de su empresa, las oportunidades para promover sus productos y apoyar las causas sociales y adopta las medidas necesarias para que sus actividades no tengan impacto negativo en sus comunidades.

Por el contrario el principal factor que muestra insatisfacción en los encuestados es que la empresa no tiene alianza con ninguna organización social que le permita desarrollar programas de beneficio para la comunidad, no planea $o$ realiza actividades sociales que involucre al personal y a sus familias y tampoco promueve el trabajo voluntario de sus trabajadores hacia la comunidad. Se puede apreciar que los niveles de satisfacción en cuanto a los indicadores de compromiso con la comunidad son de $100 \%$ y $75 \%$ con relación a la existencia de apoyo con recursos no financieros a grupos de actividades de beneficio social y la inversión de la empresa en conocer las particularidades de la comunidad e identificar sus expectativas y necesidades respectivamente.

Con respecto a los indicadores de cuidado y preservación del ambiente, en general se estableció que el nivel de los indicadores de cuidado y preservación del ambiente fue de 3,2 lo que refleja una percepción de poca satisfacción frente a cada uno de los ítems evaluados.

El nivel de satisfacción de la junta directiva está en un $50 \%$ en factores como los relacionados con la minimización de desperdicios a través de la realización de programas de auditoria verde, programas de conservación o protección ambiental. La empresa genera conciencia entre el personal sobre el cuidado del ambiente y adquiere productos y materia prima reciclables. Asímismo, se percibe un grado de insatisfacción en la percepción que tienen por la falta de programas encaminados

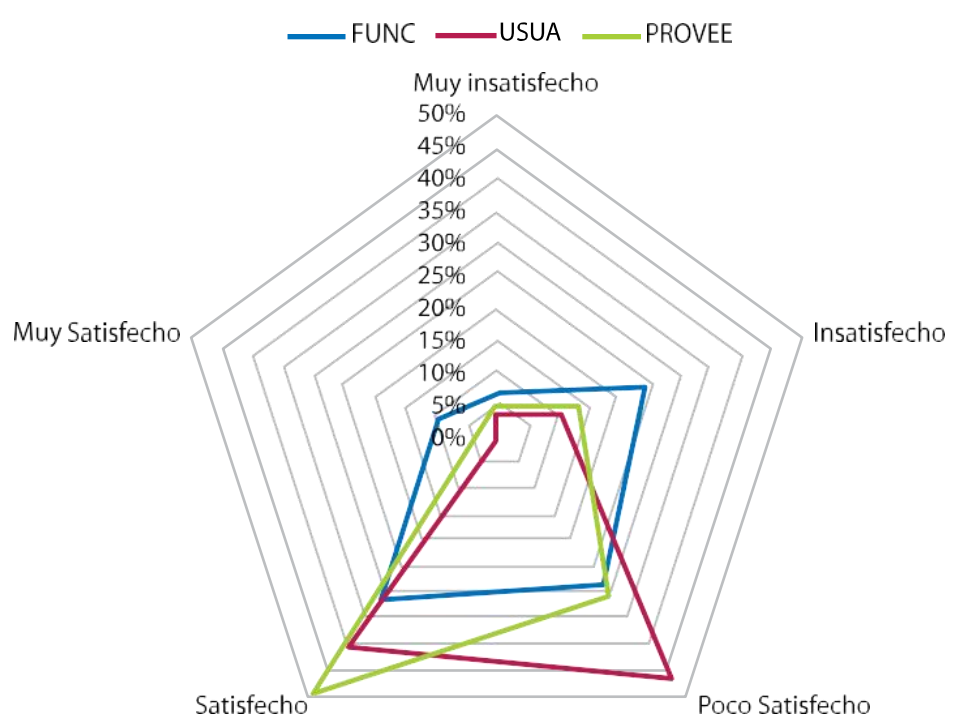

Gráfica 3. Nivel general de los Indicadores de cuidado y preservación del medio ambiente 
a convertirla en una empresa sin papel, no se perciben metas formales ni declaración de principios ambientales, mantenimiento de activos en niveles adecuados para prevenir la contaminación, como tampoco la generación de incentivos previos y reconocimientos para los empleados que sugieran alternativas de solución a los procesos de contaminación.

En relación con el cuidado y la preservación del ambiente los funcionarios muestran como punto de mayor satisfacción que la empresa cuenta con programas de recolección de residuos y reciclaje post consumo, políticas para reducir el consumo de energía eléctrica y agua en sus instalaciones, programas para el mejor aprovechamiento de recursos y minimización de desperdicios, programas encaminado a convertirla en empresa sin papel en porcentajes por encima del $50 \%$.

De la misma manera están insatisfechos y poco satisfechos en porcentajes mayores al $60 \%$ en aspectos como la falta de incentivos a empleados que sugieren alternativas en los procesos de contaminación, no se establecen compromisos explícitos con el ambiente, no se fijan estándares ni metas formales, no destina una partida del presupuesto anual para programas de conservación o protección ambiental, como tampoco para la utilización de criterios am- bientales para la selección de proveedores y además no detalla información sobre los daños ambientales ocasionados como resultado del uso de productos y/o servicios prestados.

Manifiestan en la categoría de muyinsatisfecho con $16.7 \%$ lafaltadeaplicacióndealgunanorma o certificación ambiental tipo ISO 14000(7), industria limpia u otra. Los usuarios por su parte manifestaron en cuanto a indicadores de cuidado y preservación del ambiente estar satisfechos en un $45 \%$ en aspectos como: la realización de acciones que generen conciencia de cuidado del ambiente entre el personal y su familia, y la realización de programas encaminados a convertirla en una empresa sin papel. Igualmente la insatisfacción más notoria con porcentajes de $53 \%$ corresponde a que la empresa adquiere productos y materia prima reciclables minimizando el uso de materiales no degradables, estos aspectos se evidencian en la Gráfica 4.

En cuanto a los Indicadores del cuidado y preservación del ambiente los altos niveles de satisfacción corresponden a aspectos relacionados con destinar una parte del presupuesto anual de la empresa para la conservación o protección ambiental, realizar acciones que generen conciencia sobre el cuidado del medio y contar con programas

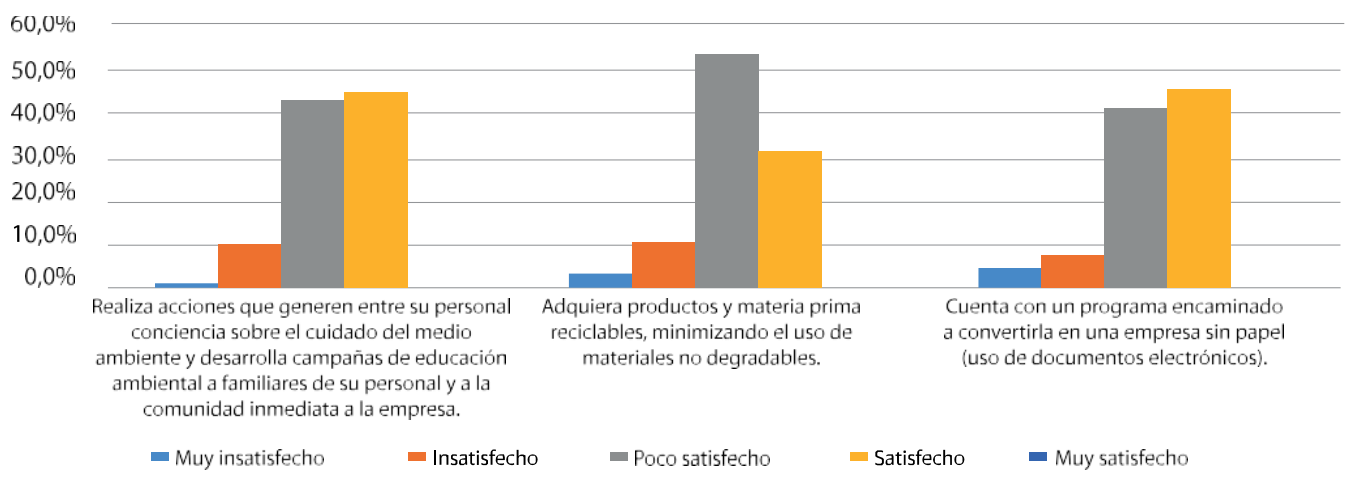

Gráfica 4. Indicadores de cuidado y preservación del medio ambiente - Usuarios 
de recolección de residuos. Mientras que los niveles de insatisfacción son de $25 \%$ y $50 \%$ relacionado con normas de certificación ambiental, e información detallada del destino de sus productos y o servicios respectivamente.

\section{DISCUSIÓN}

Encuantoalascaracterísticassociodemográficas tal ycomo lomuestra las gráficas 1 y 2 , se observa que en su mayoría en un $56 \%$ los encuestados son de sexo femenino tanto en los usuarios como en los funcionarios. Lo anterior nos indica que la Empresa Social del Estado (E.S.E) cumple con la politica nacional de inclusion donde se le da cumplimiento y se previenen las diferentes formas de discriminación, inequidad y exclusión de las mujeres en el campo laboral (8), promoviendo con ello la participación en el mismo, tal como lo establece ademas se abstiene de contratar a menores de edad, y se evidencia que solo contrata personas en edad productiva.

Los indicadores de calidad de vida mostraron como mayor fortaleza de acuerdo a la gráfica 3, la empresa fomenta y estimula el trabajo en equipo siendo este aspecto de gran relevancia en la organización. Alcaraz (9), explica que el trabajo en equipo ha ganado importancia dentro de las empresas, donde hoy en día se ha vuelto una necesidad debido a las exigencias de calidad, eficiencia y eficacia así como la competencia que existe dentro de la sociedad y es determinante dentro del sector empresarial.

La modernidad de hoy demanda un aprovechamiento y ahorro de recursos, así como la calidad de trabajo y la eficiencia en la innovación. Todo esto no lo puede lograr un solo individuo, el alcanzar y mantener el éxito en las empresas requiere de talentos que no se encuentran en una sola persona por lo que las nuevas estructuras de la empresa requiere de una interacción entre los individuos lo que se logrará con una actitud de cooperación.
Sin embargo, lo anterior genera motivación en el personal, refleja mayor liderazgo, se respetan las líneas de autoridad, lo cual mejor la competitividad empresarial, sin dejar de lado que "tendrá un impacto en la trayectoria profesional de los empleados a través de las políticas de personal que permita establecer", afirma Jin Li, profesor adjunto de estrategia en la Kellogg School. Y si bien la posibilidad de obtener un ascenso es de gran ventaja para los empleados y un incentivo para mantenerlos fieles a su empleador, la falta de posibilidades de ascenso impele a los más competentes a buscar trabajo en otro lugar.

El grado de satisfacción más alto de los funcionarios obedece a la seguridad y salud en el lugar de trabajo proporcionada por la empresa. De acuerdo con el Decreto 1443 de 2014 (10), las principales características de la política de seguridad en el trabajo, deben mostrar el ajuste a las necesidades de la empresa en donde intervengan los trabajadores y a su vez el empleador a fin de conocer el entorno y los posibles factores de riesgo existentes, intervenirlos $u$ orientar acciones de prevención que permitan a su vez identificar hallazgos y formular planes de mejoramiento a fin de que los procesos, procedimientos y tareas maximicen el buen desempeño de la entidad.

A su vez el mayor grado de insatisfacción corresponde a la falta de programas especiales para la inclusión de grupos específicos y de estímulo en la generación de ideas decisiones y creatividad a favor de la organización, lo que es de vital importancia teniendo en cuenta que la creatividad es la base de la innovación que nos permite aprovechar estos conocimientos, así como eliminar el miedo al fracaso y estar atento a cambios del mercado (11).

La opinión de los proveedores manifiesta que en la empresa se garantiza la no discriminación por motivo de origen étnico, género o posición social etc. Lo anterior representa una ventaja 
para la organización teniendo en cuenta que estos derechos se encuentran establecidos en la Declaración Universal de los Derechos Humanos (12) en sus artículos 1, 2, 7 y 23. Y que la igualdad de oportunidades y de trato permite a todas las personas desarrollar plenamente sus talentos y calificaciones de conformidad con sus aspiraciones y preferencias, y gozar de igualdad de acceso al empleo y de igualdad respecto de las condiciones de trabajo.

En cuanto a los indicadores de compromiso con la comunidad se puede verificar que como punto de menor satisfacción y oportunidad de mejora están aspectos relacionados con: políticas de apoyos sociales y programas de beneficio social, o de compromiso explícito con el ambiente.

Es importante que la empresa se vincule con las comunidades del entorno porque le permite aportar en la solución de los problemas sociales y mostrar un gran impacto en la inversión social, ello implica mucho más que otorgar donativos es mejorar la calidad vida de cada uno de los miembros de las comunidades, estas acciones generan beneficios a la organización e incrementan la moral el compromiso y la retención en el desempeño de los trabajadores, desarrollan sus habilidades y fortalecen la imagen de la empresa, por lo tanto incrementan la lealtad y preferencia de los consumidores (13). El mayor punto de satisfacción para los funcionarios en cuanto a los indicadores de compromiso con la comunidad es que la empresa responde a las expectativas que ésta última tiene de la misma e interviene para satisfacer sus necesidades. Lo anterior es de vital importancia según la Fundación Empresarial para la Acción Social (14), teniendo en cuenta que el cliente externo es la razón de ser de la empresa y que la satisfacción de los mismos significa permitir el bienestar personal y profesional de los funcionarios, esto incluye el cumplimiento de la obligación principal del empleador de proporcionar un entorno de trabajo seguro ayudar a alcanzar metas profesionales, apoyando sus capacidades aptitudes talentos y conocimientos.

Se observó, que en un $100 \%$ los proveedores tienen la percepción de que la empresa adopta las medidas necesarias para garantizar que sus actividades no tengan impacto negativo sobre la comunidad donde las desarrolla. Lo anterior apunta a que una empresa socialmente responsable es consciente de su capacidad de impacto en su entorno y estableciendo cauces de diálogo y cooperación con él, identifica estos impactos e intenta implementar mecanismos para minimizar o compensar los negativos $\mathrm{y}$ potenciar los positivos. Este compromiso con su entorno social cercano crea vínculos muy estrechos entre la empresa y la comunidad de la que forma parte, que repercutirán positivamente en la organización tanto por la identificación y compromiso de los miembros de esa comunidad que la empresa incorpore como trabajadores como por la especial atención que cabe esperar le brinden las administraciones que representan a la misma.

En cuanto a los indicadores de cuidado y preservación del medio ambiente el punto máximo de satisfacción de funcionarios corresponde a aspectos como que la empresa ha sido encaminada a convertirse en una empresa sin papel, lo que resalta una amigabilidad con el ambiente, esto se conoce con el nombre de "buenas practicas".

La aplicación de Buenas Prácticas exige comunicación y motivación a los empleados y una clara delimitación de responsabilidades interna para el seguimiento. Estas mejoras ayudarán a la empresa a ser más productiva en el largo plazo.

La apreciación de los usuarios, refleja entonces que una oportunidad de mejora de la empresa es la de adquirir productos y materia prima reciclable, teniendo en cuenta que reciclar 
es importante ya que se puede salvar grandes cantidades de recursos naturales no renovables cuando en los procesos de producción se utilizan materiales reciclados. Al reciclar se producen una serie de ventajas y beneficios que de alguna manera u otra favorecen al ser vivo, ya que reciclando se pueden salvar cantidades de recursos, como los árboles, también reduce el gasto de energía, todo eso ayuda básicamente a disminuir gran parte de la contaminación global. En la gráfica 13, se observa la poca satisfaccion en cuanto a los criterios ambientales utilizados para la selección de sus proveedores y el mayor nivel de satisfaccion en lo relacioinado con la existencia de programas de recoleccion de residuos reiclaje post-consumo.

Con respecto a lo anterior, el Ministerio de Ambiente y Desarrollo Sostenible (15) viene adelantando una estrategia dirigida a promover la gestión ambientalmente adecuada de los residuos posconsumo con el fin que sean sometidos a sistemas de gestión diferencial y evitar que la disposición final se realice de manera conjunta con los residuos de origen doméstico. Dicha estrategia involucra, como elemento fundamental, el concepto de responsabilidad extendida del productor, en el cual los fabricantes e importadores de productos son responsables de establecer canales de devolución de residuos posconsumo, a través de los cuales los consumidores puedan devolver dichos productos cuando estos se convierten en residuos.

En desarrollo de lo anterior, el Ministerio de Ambiente, Vivienda y Desarrollo Territorial, hoy Ministerio de Ambiente y Desarrollo Sostenible, ha expedido regulación para los sectores de plaguicidas, medicamentos, baterías plomo ácido, pilas y/o acumuladores, llantas, bombillas y computadores y/o periféricos. Así mismo, el Ministerio ha desarrollado estrategias voluntarias a través de alianzas y acuerdos de concertación establecidos con los fabricantes e importadores, para la recolección y gestión ambientalmente adecuada de celulares y equipos de refrigeración en desuso.

Finalmente a nivel general y teniendo en cuenta la valoración de la percepción en cada grupo de indicadores, el nivel de Responsabilidad Social de la institución es de 3,0, lo cual se clasifica como poco satisfactorio, de acuerdo como se ilustra en la gráfica 5 .

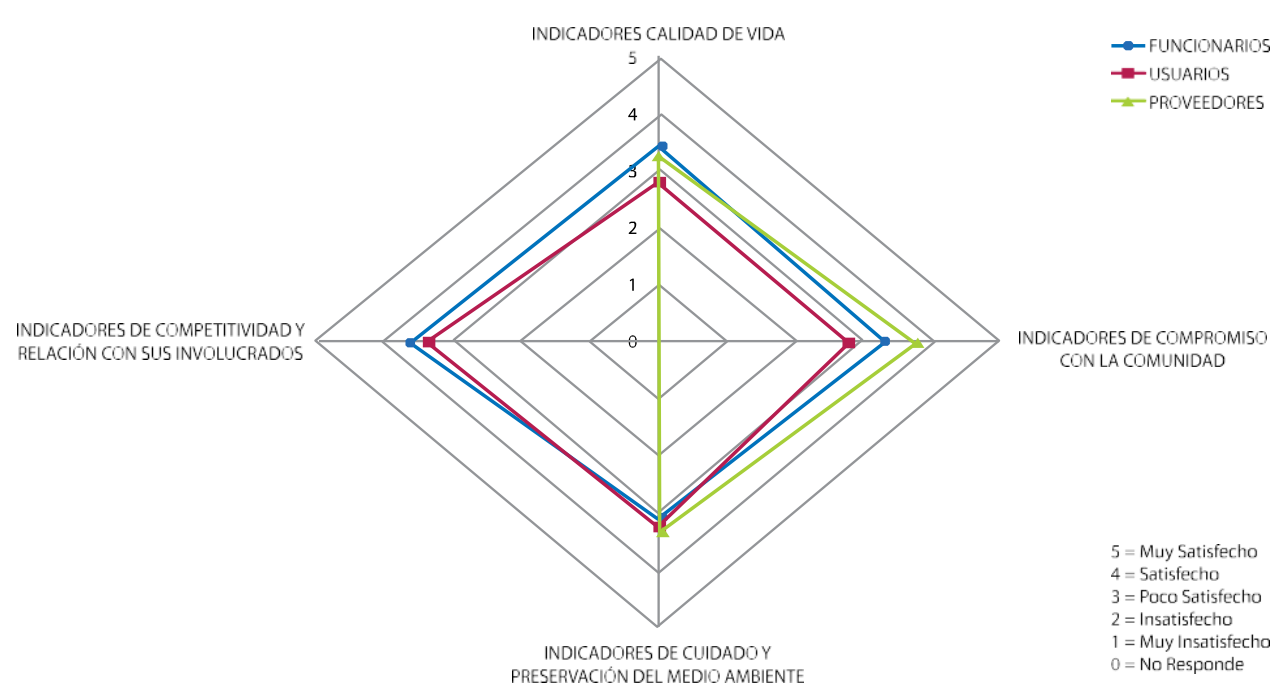

Gráfica 5. Nivel general de Indicadores de responsabilidad social empresarial 


\section{CONCLUSIONES}

En relación a las actuales condiciones en las cuales se encuentra la Empresa Social del Estado con respecto a la percepción que tienen sus principales grupos de interés frente al nivel de Responsabilidad Social, se puede señalar inicialmente que su nivel es poca satisfactoria ya que en la organización se realizan acciones aisladas, las cuales no se encuentran insertadas o articuladas en un plan institucional que direccione este tipo de responsabilidad al interior de la empresa, por ello se recomienda además de definir un plan estratégico para establecer una planeación formal de la RSE, con la participación activa de los grupos de interés y la información de los logros a través de medios efectivos de comunicación.

Para cada grupo de indicadores, se concluye:

1. En cuanto a los indicadores de calidad de vida de la empresa se encontró un nivel de percepción de poca satisfacción, en el que se puede identificar como fortalezas la percepción que tienen la junta directiva, funcionarios, los usuarios y proveedores en cuanto al fomento del trabajo en equipo, mecanismos que favorecen el desarrollo, garantizar la no discriminación, las políticas de asociación, el incremento de número de empleos femeninos, manejo con respeto el derecho a liquidaciones y despidos, realizar capacitaciones continuas a los empleados, oficina de atención al cliente y mecanismos para conocer el grado de satisfacción de los mismos, políticas de no discriminación por ningún motivo de origen étnico, género, o posición social.

Asímismo, los grupos de interés perciben como oportunidades de mejora los factores relacionados con la falta de un plan para el desarrollo de sus empleados y la calidad de vida de las familias y prestaciones superiores a la ley, poco ascenso en empleos femeninos a posiciones ejecutivas, falta de esquemas de trabajos flexibles y de programas para estimular la generación de ideas, decisiones y creatividad y poco estímulo a las oportunidades de trabajo voluntario para los empleados en proyectos sociales, disposición de realizar investigaciones de clima organizacional y desarrollar campañas periódicas para informar e identificar áreas que requieran atención, desconocimiento del código de ética y gestión de la empresa en cuanto a reclamaciones y sugerencias para darles respuestas a las mismas.

2. De acuerdo a los indicadores de compromiso con la comunidad, se halló un nivel de percepción de poca satisfacción en el que los grupos de interés manifiestan satisfacción en aspectos como que la empresa invierte en conocer las expectativas y necesidades de la comunidad, dando respuesta las mismas y estableciendo canales de comunicaciones entre sí, involucra de igual manera a las familias y se promueve el trabajo voluntario, se buscan oportunidades para promover productos $y / o$ servicios $y$ se adoptan medidas necesarias para garantizar que las actividades a realizar no tengan impacto negativo sobre la comunidad, la existencia de apoyo con recursos no financieros a grupos de actividades de beneficio social y la inversión de la empresa en conocer las particularidades de la comunidad e identificar sus expectativas y necesidades respectivamente.

De igual manera de acuerdo a la opinión de los grupos encuestados son oportunidades de mejora los aspectos que se relacionan con, contar con una instancia o fundación responsable de la gestión de donativos o conducir la vinculación con la comunidad para la realización de actividades públicas cívicas o de beneficio social por lo que contribuye al bienestar económico y social de las comunidades en las que opera. 
3. En cuanto a los indicadores de cuidado y preservación del ambiente, existe un nivel de percepción de poca satisfacción en el que se puede identificar como fortalezas la percepción que tienen la junta directiva, funcionarios, los usuarios y proveedores en cuanto a la minimización de desperdicios a través de la realización de programas de auditoria verde, así como programas de conservación o protección ambiental, adquisición de productos y materia prima reciclables, con programas de recolección de residuos y reciclaje post consumo, políticas para reducir el consumo de energía eléctrica y agua en sus instalaciones, programas para el mejor aprovechamiento de recursos y minimización de desperdicios, programas encaminado a convertirla en empresa sin papel, realización de acciones que generen conciencia de cuidado del ambiente entre el personal y su familia, destinar una parte del presupuesto anual de la empresa para la conservación o protección ambiental, realizar acciones que generen conciencia sobre el cuidado del medio.

En cuanto a las oportunidades de mejora que se pueden percibir de acuerdo a la opinión de los grupos de interés se destacan los aspectos relacionados con las normas de certificación ambiental, e información detallada del destino de sus productos y o servicios respectivamente, no se perciben metas formales ni declaración de principios ambientales para prevenir la contaminación, como tampoco la generación de incentivos previos y reconocimientos para los empleados que sugieran alternativas a los procesos de contaminación, no se establecen compromisos explícitos con el medio ambiente como tampoco se fijan estándares ni metas formales así como que la empresa no detalla información sobre los daños ambientales ocasionados como resultado del uso de productos y/o servicios prestados.
4. En el indicador de competitividad y relación con los involucrados los grupos de interés encuestados, coinciden en estar cumpliendo las obligaciones fiscales y los mecanismos para sugerencias, quejas y reclamos $y$ la existencia de políticas, mecanismos y procedimientos para el seguimiento de las sucursales contra la corrupción, contar con una política de equidad para la selección y pago de sus proveedores, mecanismos que garanticen la congruencia entre calidad $y$ precio, sistemas para conocer, entender y atender las necesidades e inquietudes de sus clientes, políticas de trato a sus clientes que garanticen la honradez en todas sus transacciones y que ofrezcan atención y solución a todas sus reclamaciones, manejo de mensajes promocionales y publicitarios, sistema de mejoramiento continuo en aspectos como calidad y productividad, programas para desarrollar el liderazgo asertivo, mecanismos a través de los cuales los empleados de mayor experiencia compartan sus conocimientos, política de respeto a los competidores e informar interna y externamente los logros y retos de la empresa.

Asímismo, se encontró como una oportunidad de mejora lo relacionado con la disposición de programas de apoyo y cooperación para el desarrollo de sus proveedores en particular de los locales y mecanismos que garanticen la congruencia entre calidad y precio más allá del mercado, también la falta de programas para desarrollar el liderazgo asertivo - proactivo en las jefaturas de los mandos medios.

\section{RECOMENDACIONES}

De acuerdo con los objetivos planteados, los resultados y las conclusiones, el grupo investigador propone las siguientes estrategias con el único fin de mejorar el nivel de responsabilidad social empresarial que 
actualmente presenta la Empresa Social del Estado ESE Hospital Local de Montelibano:

1. Realizar al interior de la empresa una evaluación de la plataforma estratégica, planes, programas, proyectos y políticas a fin de ajustarlas e introducir elementos operativos de responsabilidad social en los cuatro indicadores evaluados, que se articulen y no impliquen mayor inversión en su implementación de acuerdo al plan de mejoramiento adjunto.

2. En cuanto a los indicadores de calidad de vida se sugiere la socialización del código de ética con el fin de sensibilizar a los diferentes grupos de interés sobre los valores que deben liderar sus relaciones, realizar un plan para el desarrollo de calidad de vida de sus empleados, familias, proveedores y usuarios que incluya lineamientos sobre la inclusión y contratación de grupos especificos, trabajos flexibles, voluntario, proyectos sociales y evaluación de las satisfacción de los grupos.

3. Encuantoalasactividadesderesponsabilidad social empresarial en el compromiso con la comunidad se sugiere crear una fundación o sistema responsable de la planeación, otorgamiento y seguimiento de donaciones, apoyo con recursos no financiero (personal, equipos, servicio) a grupos organizados de la comunidad para la realización de actividades públicas, cívicas o de beneficio social, actividades de diagnóstico e intervención de las necesidades de las comunidades que permitan realizar talleres, capacitaciones, donde la comunidad y la empresa se integren y participen del desarrollo mutuo.

4. Con respecto al cuidado y preservación del medio ambiente la empresa debe establecer un sistema de incentivos, premios y reconocimientos a los funcionarios, administrativos y asistenciales que sugieran alternativas de descontaminación o aprovechamiento de recursos en los procesos empresariales con lo cual se estimularía la cultura de la protección y el reciclaje, en campañas como creación y diseño de árboles navideños con bolsas de dextrosas, talleres de artes y pinturas con tapitas de medicamentos etc. Además se sugiere asignar una partida del presupuesto anual a programas de preservación ambiental y a la implementación de la norma ISO 14000, realizar actividades para el mantenimiento preventivo de sus activos con el fin de mantener niveles bajos de contaminación. Diseñar e implementar el uso de comunicación electrónica a fin de minimizar la utilización del papel.

5. En cuanto a la competitividad y su relación con sus involucrados la empresa debe establecerprogramasdeapoyoycooperación para el desarrollo de sus proveedores, establecer mecanismos de sistematización del conocimiento para apoyo al relevo generacional en la que los empleados de mayor edad compartan sus conocimientos con los de menos experiencia.

Así mismo establecer políticas, mecanismos y procedimientos que promuevan la honestidad en sus proveedores $y$ sus funcionarios.

Realizar un plan de seguimiento y evaluación que permita medir los avances a través del balance social, la auditoria interna, auditoria verde, análisis financiero y medir los resultados de las metas propuestas después de la implementación de las estrategias de mejora propuestas. 


\section{REFERENCIAS}

1. Echaide J. Responsabilidad Social Empresaria: un sobrevuelo por la norma ISO 26000. Lan Harremanak. Revista de Relaciones Laborales, (20-21).2011

2. Casino Ch, Morales M. Responsabilidad social empresarial: Ed. 2,diciembre de 2008

3. Carnegie A. empresario y filántropo estadounidense oriundo de Escocia.

4. Vélez Pareja I. Decisiones de inversión para la valoración financiera de proyectos $y$ empresas, 2001, p. 30.

5. Córdoba A. El reto de la gestión empresarial. Barcelona: Deusto. Pág. 96. 2005

6. Del Empresariado Chihuahuense, F. RSE: Instrumento de autodiagnóstico para las empresas. Ciudad Juárez: FECHAC.

7. De Oliveira, M. SA 8000: Modelo ISO 9000 aplicado à responsabilidad social. Qualitymark Editora Ltda. 2003.

8. Ministerio Del Trabajo. Programa nacional de equidad laboral con enfoque diferencial de género. 2014

9. Alacaraz, R. El emprendedor de éxito. Tercera edición. McGraw Hill. México

10. Ministerio Del Trabajo. Decreto 1443. Colombia, 2014

11. Cortina A. Construir Confianza.
Ética de la empresa en la sociedad de la información y las comunicaciones. Madrid: Editorial Trotta. 226 p.2003

12. Anónimo, A. Declaración Universal de los Derechos Humanos. Dialéctica Libertadora, (1), 161-172. 2014.

13. Fundación del empresario por la Responsabilidad Social México. Pág. 10

14. FUNDEMAS, "La Empresa Amigable con el Medio Ambiente" disponible en: http://www.bibliotecavirtual.info /wp- content/uploads/2013/11/esmanual-del- participante-empresamedio-ambiente.pdf

15. Ministerio del medio ambiente y desarrollo sostenible, programa posconsumo de residuos. Disponible: https://www.minambiente.gov .col index.php/component/conte nt/ article?id=28:plantillaasuntos - ambientales-y-sectorial-yurbana

16. Banco Interamericano del Desarrollo. III Conferencia Interamericana sobre R.S.E. La Responsabilidad Social de las Empresas. Santiago de Chile. 2005. pág. 4. En: www. cruzroja.org/od/redcamp/eventos 